\title{
Application d'un modèle de pénétration de la lumière à une jeune plantation de hêtre avec abri latéral*
}

\author{
I Planchais ${ }^{1,2}$, JY Pontailler ${ }^{* *}$ \\ 'Laboratoire d'écologie végétale, CNRS-Ura 2154, Bât 362, université Paris-XI, 91405 Orsay cedex ; \\ ${ }^{2}$ Département des recherches techniques, Office national des forêts, boulevard de Constance, \\ 77300 Fontainebleau cedex, France
}

(Reçu le 5 janvier 1996 ; accepté le 11 mars 1996)

Summary - A radiative transfer model applied to a young beech plantation. A radiative transfer model developed for row crops (Sinoquet, INRA-Clermont-Ferrand, France) was applied to a young beech row plantation, partly sheltered by a mature forest. The leaf area index of the forest was estimated by a LAI 2000 PCA (plant canopy analyser), and the leaf area uniformly distributed within the canopy. In each row, 15 beech trees were measured to establish the main dimensions of the mean tree and its crown. The rows were then modelled as a series of mean individual plants, whose leaf area was estimated from an allometric relationship with the diameter of the trees, and then uniformly distributed within the crowns. The test of the model was based on radiation measurements in the PAR waveband below and above the rows. With regard to transmitted radiation above the five rows, the outputs of the model were in close agreement with the measurements. In spite of large discrepancies in the estimation of transmitted radiation at ground level, global results suggest that the model correctly approximates light distribution inside the plantation. By testing a few simple hypotheses, it was shown that the fine structure of young beech trees (spatial variation in leaf area density, clumping) needs to be further investigated. This approach, suited to account for any canopy structure, appears to be a useful predictive tool for assessing the radiation environment within specific discontinuous canopies.

light / Fagus sylvatica / radiative transfer model / row plantation

Résumé - Un modèle de pénétration de la lumière dans les cultures en rang, développé par Sinoquet (Inra Clermont-Ferrand), a été appliqué de manière originale à une jeune plantation de hêtres abritée latéralement par un couvert forestier adulte. L'indice de surface foliaire (LAI) de l'abri latéral a été déterminé à l'aide d'un LAI 2000 PCA (plant canopy analyser), et cette surface

\footnotetext{
* Cet article présente des résultats acquis dans le cadre d'un travail de DEA réalisé au laboratoire d'écologie végétale (université Paris-XI)

** Correspondance et tirés à part
}

Tél. : (33) 0169157961 ; fax : (33) 0169157238 ; courriel : jean-yves.pontailler@eco.u-psud.fr 
foliaire ensuite répartie uniformément dans le volume occupé par la forêt. Les cinq lignes de la plantation sont représentées de manière simplifiée par une succession d'individus moyens, dont les dimensions sont issues de mesures morphologiques réalisées sur 15 arbres par ligne, et dont la surface foliaire est estimée par une relation allométrique utilisant le diamètre des plants. Le test du modèle a été réalisé par la mesure du rayonnement transmis au sommet et au bas des lignes de la plantation. Il montre que le modèle rend compte de façon très satisfaisante de l'ombrage latéral dû à la forêt, alors que des écarts plus importants s'observent au sol. Le test de quelques hypothèses simples concernant la répartition et l'agencement des feuilles dans les houppiers montre que la description de la structure des jeunes hêtres demande à être approfondie. À l'issue de cette première approche, il semble que la qualité des estimations apportées par le modèle Sinoquet en fasse un outil d'un grand intérêt pour la caractérisation du microclimat lumineux de certains couverts forestiers particulièrement complexes.

lumière / Fagus sylvatica / modèles de transferts radiatifs / plantation avec abri latéral

\section{INTRODUCTION}

Parmi les facteurs du milieu influençant le développement des végétaux, la lumière joue un rôle déterminant, sur leur bilan carboné comme sur leurs pertes en eau. Dans le cas plus particulier des jeunes recrûs forestiers, la répartition inégale de la lumière au sein du couvert conduit très tôt, au gré des relations de compétition entre individus voisins, à une différenciation morphologique des arbres, puis à une évolution structurale du peuplement déterminante pour son avenir. Par ailleurs, la lumière étant de loin le facteur du milieu le mieux maîtrisé par le praticien lors des opérations sylvicoles, il est important de pouvoir quantifier cette répartition inégale de la lumière dans les jeunes couverts forestiers.

De nombreux modèles mathématiques ont été développés afin de simuler la distribution spatiale de la lumière et son interception par les végétaux. Toutefois, la majorité d'entre eux font l'hypothèse de l'homogénéité du couvert dans le plan horizontal, et ne permettent donc pas d'étudier le cas des structures discontinues pourtant fréquemment rencontrées en forêt : jeunes régénérations ou plantations de faible densité, jeunes peuplements en trouées ou sous abri latéral, futaie jardinée...
Quelques modèles de pénétration du rayonnement sont toutefois adaptés au cas des structures discontinues. Ils peuvent être classés en deux groupes (Lemeur et Blad, 1974 ; Sinoquet, 1993) :

- les modèles géométriques, qui décrivent le couvert par une série de formes géométriques bien définies (cylindre long pour une ligne d'arbres d'un verger, ellipsoïde pour un houppier), régulièrement disposées, et dont les enveloppes constituent le lieu des échanges radiatifs. Si celles-ci sont supposées opaques (Jackson et Palmer, 1972), le calcul du rayonnement intercepté repose alors uniquement sur des considérations d'ordre géométrique (calcul de l'ombre portée d'un ligne sur l'autre).

- les modèles statistiques, qui fondent leur description du couvert sur une distribution statistique décrivant l'arrangement des feuilles dans l'espace, sans faire référence à leur position exacte dans la canopée. L'atténuation du rayonnement due à l'interception par les feuilles est calculée par la loi de Beer-Lambert. En raisonnant dans une direction donnée $\theta$ du rayonnement incident $(\theta$ est l'angle zénithal), la loi de Beer-Lambert s'écrit :

$$
f_{1}=\exp (-\operatorname{LAD} \times \Omega \times G(\theta) \times S(\theta))
$$


où $f_{t}$ est la fraction de trouée, c'est-à-dire la proportion du rayonnement incident (audessus du couvert) qui parvient à l'endroit considéré (au sein du couvert); LAD est la densité de surface foliaire (surface des feuilles présentes dans l'unité de volume, en $\mathrm{m}^{2} / \mathrm{m}^{3}$ ) le long du trajet lumineux ; $\Omega$ est appelé paramètre d'agencement : il permet de prendre en compte la diminution relative d'interception due à l'agrégation des feuilles $(\Omega=1$ si la répartition des feuilles est aléatoire ; $\Omega<1$ s'il y a agrégation) ; $S(\theta)$ est la longueur du trajet lumineux dans la canopée $(\mathrm{m}) ; G(\theta)$ est le coefficient de projection de l'unité de surface foliaire dans le plan normal au rayonnement incident (rapport de la surface projetée à la surface réelle) : $\mathrm{G}(\theta)$ dépend donc de l'inclinaison des feuilles.

Afin d'être rendue applicable au cas des couverts discontinus, cette approche statistique nécessite une discrétisation (subdivision de l'espace en volumes homogènes). Certains modèles (Charles-Edwards et Thorpe, 1976 ; Wang et Jarvis, 1990) attribuent à un individu isolé, ou à un rang, une enveloppe de forme définie à l'intérieur de laquelle densité foliaire et distribution d'inclinaison foliaire sont supposées uniformes. Une autre possibilité consiste à discrétiser l'espace en « cellules » parallépipédiques (Kimes et Kirchner, 1982 ; Sinoquet, 1988). Le test de tels modèles appliqués au cas de couverts forestiers discontinus a rarement été réalisé du fait de la complexité des structures à décrire.

Le modèle Sinoquet s'applique aux couverts végétaux présentant une structure périodique : il suffit alors d'en décrire la maille élémentaire, qui couvre une période de la structure. Sur cette maille élémentaire, l'espace compris entre le sol et le sommet du couvert est structuré en niveaux horizontaux, puis en tranches verticales selon deux directions perpendiculaires, définissant ainsi un certain nombre de cellules parallépipédiques.
Chaque cellule est définie par sa position, le nom de la ou des espèces qu'elle contient éventuellement, et, pour chaque espèce, une densité de surface foliaire et une distribution d'inclinaison foliaire. La répartition des feuilles est supposée aléatoire.

Pour chaque orientation du rayonnement incident, le modèle détermine la succession des cellules traversées. L'extinction progressive du rayonnement est alors calculée conformément à l'équation 1 , en appliquant la loi de Beer-Lambert à chacune d'elles. Enfïn, la voûte céleste est divisée en secteurs d'angles solides, et les flux provenant de l'ensemble de l'hémisphère sont calculés par intégration numérique des flux directionnels.

Les principaux objectifs de cet article seront donc :

- i) de tester un modèle statistique de pénétration de la lumière dans le cas d'une jeune plantation de hêtres sous abri latéral, structure marquée par une répartition très contrastée de la lumière. Parmi les différents modèles existants, nous avons retenu le modèle statistique de Sinoquet (1988), adapté au cas des plantations en rang, et qui permet aisément de prendre en compte la géométrie d'une plantation sous abri latéral ;

- ii) de caractériser le microclimat lumineux associé à chaque ligne de la plantation ;

-iii) de discuter de l'intérêt et des difficultés posées par une telle approche.

\section{MATERIEL ET MÉTHODES}

\section{Site}

Le site retenu est situé en forêt domaniale de Compiègne (parcelle XI26; $49^{\circ} 18 \mathrm{~N}, 2^{\circ} 42 \mathrm{E}$ ) sur un sol brun lessivé sur sable épais. Une jeune plantation de hêtres, en bandes orientées SWNE, se développe sous l'abri latéral d'une chênaie-charmaie (taillis-sous-futaie vieilli d'une 

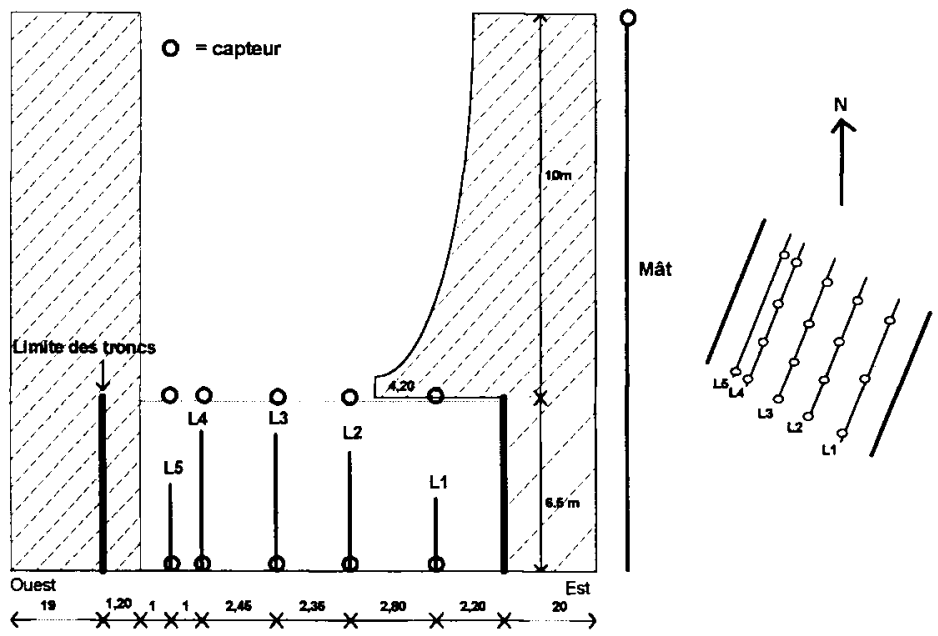

Fig 1. Coupe schématique du site montrant l'abri latéral, les cinq lignes et l'emplacement des capteurs de rayonnement.

hauteur de $16,5 \mathrm{~m}$ environ) (fig 1 ). La lisière, côté est, empiète assez largement sur les lignes 1 et 2 . La zone retenue, caractérisée par une bonne homogénéité de son abri latéral ainsi que des plants sur les cinq lignes, s'étend sur une trentaine de mètres.

La plantation, réalisée en 1986, compte cinq lignes d'arbres de 11 ans d'âge (fin 1993), dont
8 années en plantation. La figure 2 présente les dimensions moyennes (diamètre et hauteur) des arbres de chaque ligne. La ligne 4 est visiblement la plus développée, dominant de peu la ligne 3. En revanche, les arbres de la ligne 2 sont significativement plus petits, en diamètre comme en hauteur. Enfin, les lignes latérales sont considérablement moins développées (mais également

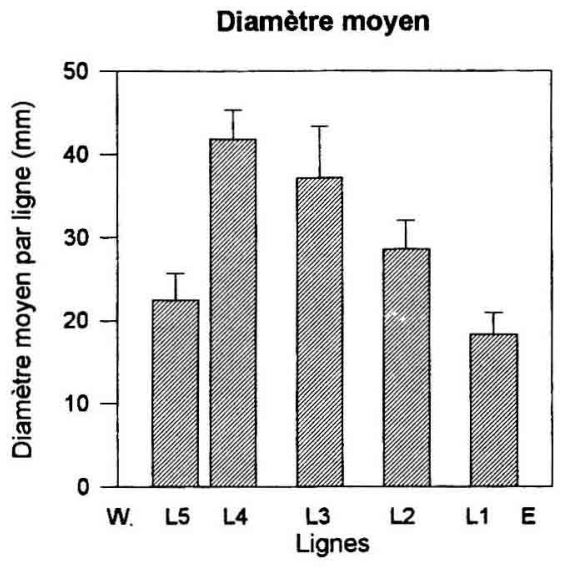

Comparaison de moyennes : test Mann-Whitney-Wilcoxon L5/L4: **; L3/L2 : *; L2/L1 : **

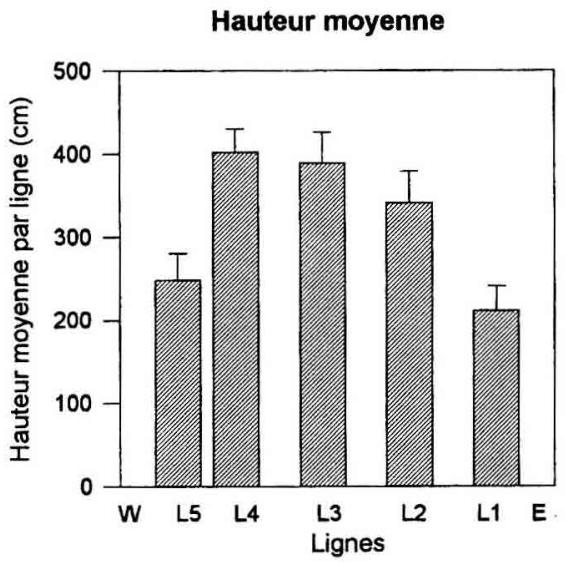

Comparaison de moyennes : test Mann-Whitney-Wicoxon L5/L4 : * L3/L2 ; * : L2/L1: ***

Fig 2. Diamètre et hauteur moyens par ligne (moyenne, intervalle de confiance à $5 \%$ ). 
plus jeunes de 2 ans, du fait de regarnis). L'espacement des plants sur les lignes centrales (L2 à L4) est de $90 \mathrm{~cm}$, et de 1,30 et $2,70 \mathrm{~m}$ respectivement pour les lignes 1 et 5 .

\section{Caractérisation de la structure du couvert}

\section{Morphologie des plants}

Sur chaque ligne, les 15 individus contigus ont fait l'objet des mesures suivantes (fig 3) : hauteur du bourgeon terminal $(\mathrm{H})$, diamètre à $20 \mathrm{~cm}$ du sol (D), hauteur de la première branche vivante $\left(\mathrm{H}_{\mathrm{bv}}\right)$, largeur du plus grand diamètre du houppier (égale à la moyenne de deux diamètres perpendiculaires de la projection du houppier) (LC), et position de ce grand diamètre $\left(\mathrm{H}_{\mathrm{LC}}\right)$. On définit ainsi les parties supérieures et inférieures du houppier.
Par ailleurs, une structuration verticale simple des houppiers a été réalisée, en distinguant trois parties fonctionnellement distinctes : P1, flèche du houppier, est constituée des deux dernières unités de croissance sur l'axe principal. Cette partie, peu ramifiée car jeune, a un rôle d'exploration du milieu. L'identification des cicatrices hivernales permet aisément d'en mesurer la hauteur. P2 correspond à la partie principale du houppier, où les axes, plus grands et plus ramifiés, supportent l'essentiel de la masse foliaire de l'arbre. Enfin, P3 est la partie inférieure de l'arbre, en phase d'élagage : plus âgée, elle se distingue par des branches plus nettement horizontales, dont l'élongation annuelle est très limitée (pousses courtes uniquement). La hauteur de chacune de ces trois parties a été précisément mesurée.

\section{Détermination de la surface foliaire des jeunes hêtres}

Elle a été réalisée par l'établissement, grâce à un échantillonnage destructif, de relations allo-

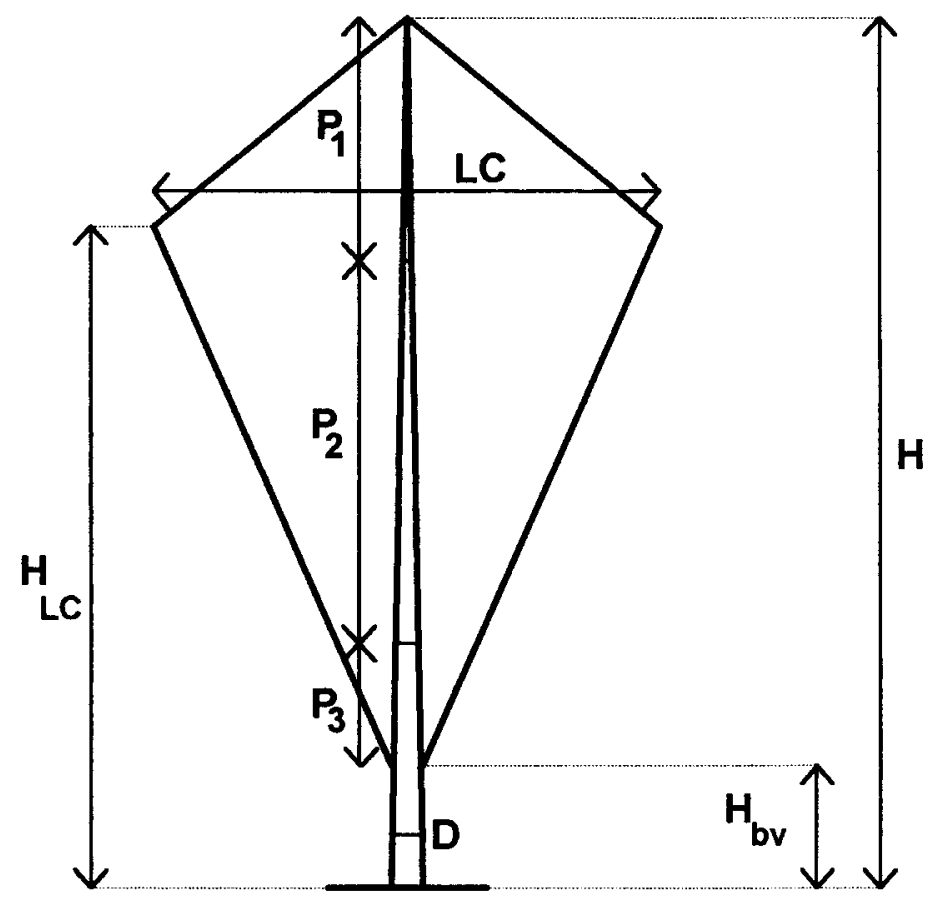

Fig 3. Caractérisation morphologique des arbres : principaux paramètres mesurés. 


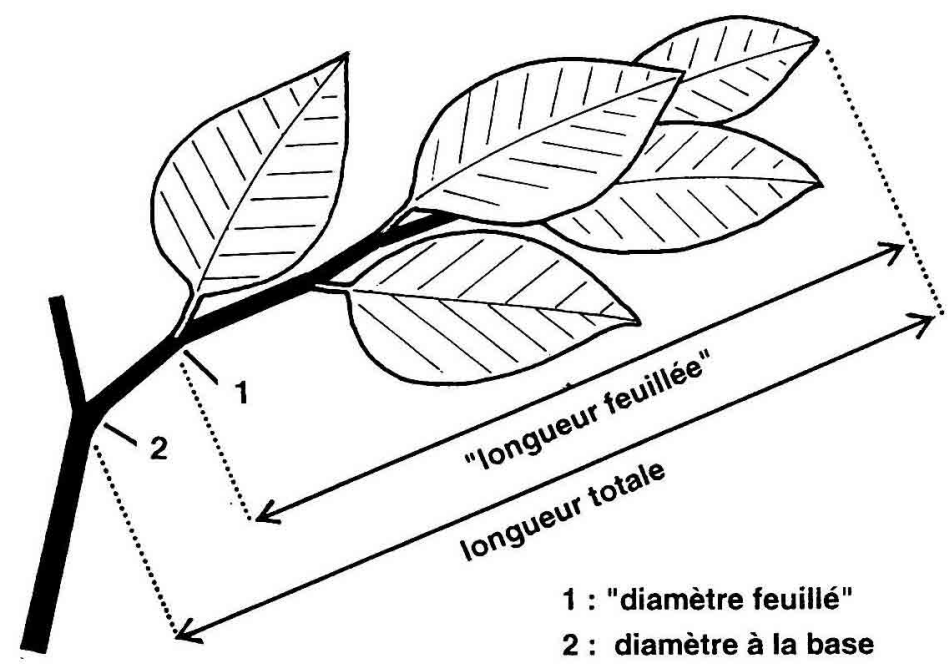

Fig 4. Paramètres utilisés pour établir la relation allométrique de la surface foliaire d'une branche.

métriques liant la surface foliaire de l'arbre à son diamètre à $20 \mathrm{~cm}$. À cette fin, quatre individus par ligne ont été coupés (soit 20 arbres en tout), dont on a précisément mesuré :

- le diamètre à $20 \mathrm{~cm}$ et la hauteur,

- le diamètre à la base, la longueur totale, le « diamètre feuillé » et la « longueur feuillée » de toutes les branches (fig 4).

On a par ailleurs collecté, sur chaque individu, une branche par tiers de houppier. La surface foliaire de chacune de ces 60 branches a été mesurée directement au planimètre (Delta- $T$ area meter, Delta-T Devices, Burwell, Royaume-Uni), de façon à établir une première relation allométrique à l'échelle de la branche. Celle-ci a ensuite permis de déterminer, par cumul des estimations de surface foliaire de toutes les branches de chaque arbre, la surface foliaire totale des 20 arbres utilisés. Une relation allométrique à l'échelle de l'arbre est finalement établie.

\section{Indice de surface foliaire de l'abri latéral}

L'indice de surface foliaire (LAI) de l'abri latéral a été déterminé à l'aide d'un LAI 2000 PCA (plant canopy analyser ; Li-Cor, Lincoln, NE, Etats-Unis), outil d'analyse des couverts végétaux qui mesure la transmission au sol du rayonnement diffus, dans cinq couronnes concentriques centrées sur le zénith. En faisant l'hypothèse d'une répartition aléatoire du feuillage, il permet de calculer l'indice de surface foliaire. Deux transects ont été réalisés sous l'abri latéral, de chaque côté de la plantation, dans des conditions de ciel couvert (fin de journée). Les valeurs de rayonnement à découvert (référence) ont été aisément acquises dans une clairière à proximité immédiate du site. L'étroitesse de l'abri latéral (bandes de $20 \mathrm{~m}$ de large) nous a contraints à utiliser un cache, de façon à restreindre l'angle de vue du capteur à un secteur entièrement occupé par la forêt. L'exploitation des données, réalisée en conservant uniquement les quatre anneaux supérieurs, a fourni pour chaque transect une valeur de l'indice foliaire de $5,5 \mathrm{~m}^{2} / \mathrm{m}^{2}$.

\section{Distribution d'inclinaison des feuilles de hêtre}

Elle a été estimée par des mesures directes (au rapporteur et fil à plomb) de l'inclinaison de 25 feuilles par tiers de houppier, et ce sur trois individus, positionnés différemment par rapport à l'axe de la plantation. Une distribution d'inclinaison en six classes de $15^{\circ} \mathrm{d}$ 'amplitude a été établie. 


\section{Mesure de rayonnement et test du modèle Sinoquet}

\section{Mesures de rayonnement}

Le test du modèle a été réalisé par la mesure du rayonnement transmis à deux niveaux dans la plantation (fig 1). Nous nous sommes intéressés à la fraction du rayonnement solaire utilisée par la photosynthèse (photosynthetically active radiation ou PAR, flux de photons entre 400 et 700 nanomètres), la végétation étant très transparente à la partie infrarouge du spectre (au-delà de 700 nanomètres). Les mesures de rayonnement ont été réalisées grâce à l'installation de 12 capteurs de PAR à l'arsénure de gallium, pourvus d'un filtre bleu (modifié d'après Pontailler, 1990) : cinq d'entre eux, fixés dans l'axe de chaque ligne sur une courte tige métallique de $20 \mathrm{~cm}$ de hauteur, mesurent le rayonnement transmis au niveau du sol. Une seconde ligne de cinq capteurs, fixés sur une corde tendue transversalement dans la plantation et maintenus parfaitement horizontaux par un contrepoids, permet la mesure du rayonnement transmis à 4,70 $\mathrm{m}$ de hauteur. Enfin, l'ouverture d'une clairière à $30 \mathrm{~m}$ du site a permis l'installation de deux capteurs à proximité du sommet du couvert, sur une perche télesco- pique de $12 \mathrm{~m}$ : I'un d'eux mesure la totalité du PAR incident et le second, équipé d'un anneau permettant de cacher le disque solaire, mesure uniquement la composante diffuse de ce rayonnement.

Ces 12 capteurs sont reliés à une centrale d'acquisition de données (CR 10, Campbell Scientific, Shepshed, Royaume-Uni et Logan, États-Unis), qui enregistre le rayonnement reçu par chaque capteur toutes les $15 \mathrm{~s}$, et mémorise les moyennes toutes les $15 \mathrm{~min}$. Ces mesures ont été effectuées en continu au cours de trois journées successives du début du mois de juin 1994. Les journées étudiées n'étant ni parfaitement ensoleillées, ni parfaitement couvertes du matin au soir, nous avons été amenés à effectuer un tri parmi les données acquises, de façon à reconstituer deux journées «type » du mois de juin (fig 5):

- une journée parfaitement ensoleillée : seules sont conservées les données où la fraction de rayonnement diffus à découvert est inférieure à $30 \%$.

- une journée parfaitement couverte, où la fraction de rayonnement diffus est supérieure à $99 \%$.

Ces journées restent partielles, car quelques plages horaires sont manquantes.

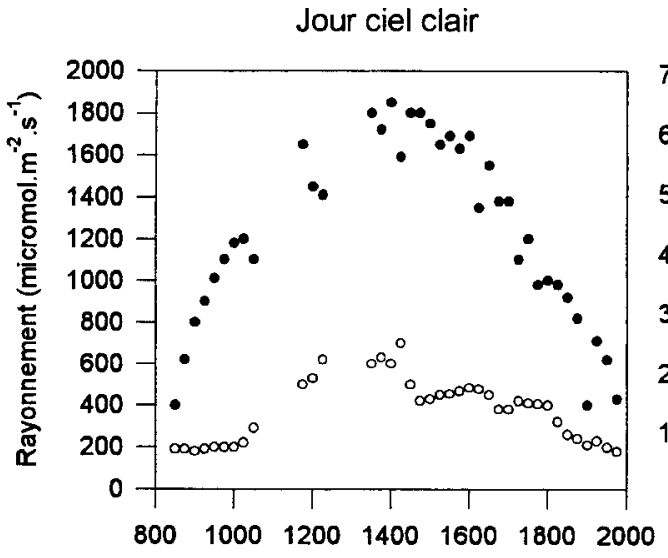

Heure légale

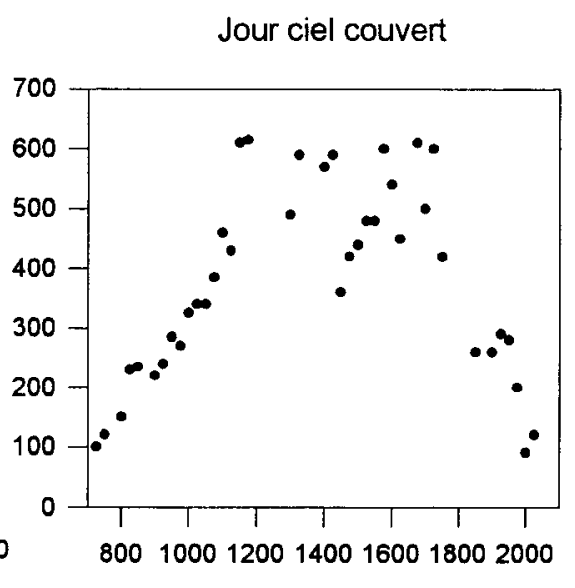

Heure légale

Fig 5. Évolution du rayonnement incident (PAR) au cours des deux journées types reconstituées. Chaque point est la moyenne du rayonnement reçu pendant les 15 min précédentes. 


\section{Modélisation du couvert}

Les cinq lignes de la plantation ont été représentées de manière simplifiée comme une succession d'individus moyens, dont les caractéristiques morphologiques (diamètre, hauteur, position et dimensions du grand diamètre du houppier) sont la moyenne des paramètres relevés par ligne. La surface foliaire de l'arbre de chaque ligne est ensuite déterminée grâce à la relation allométrique établie. La surface foliaire des jeunes hêtres a été répartie de manière uniforme au sein du volume défini pour les houppiers : des observations empiriques nous ont amenés à représenter la partie supérieure du houppier (définie par $\mathrm{H}, \mathrm{H}_{1 \mathrm{C}}$ et LC) par un cône, et la partie inférieure par un ellipsoïde. Enfin, nous avons introduit, pour chaque tiers de houppier, la distribution d'inclinaison mesurée.

L'abri latéral est constitué d'un niveau supérieur (canopée), contenant l'essentiel de la masse foliaire, et dont on a précisément mesuré la limite inférieure, voisine de $6,5 \mathrm{~m}$. Les trois quarts de la surface foliaire totale de l'abri latéral ont été répartis uniformément dans cette partie supérieure (soit entre les hauteurs de 6,5 et $16,5 \mathrm{~m}$ ), et le quart restant dans la partie basse de l'abri (contenant essentiellement branches et troncs).
On a par ailleurs utilisé une distribution d'inclinaison planophile pour l'ensemble de l'abri. Les caractéristiques de la lisière empiétant sur la plantation (côté est) ont été précisément mesurées : l'écart entre la lisière (limite des houppiers) et la limite des troncs, la longueur moyenne des branches empiétant sur les lignes 1 et 2, sont respectées dans la modélisation du couvert (fig 6). La même densité de surface foliaire que celle utilisée pour la canopée y a été introduite.

Afin de bien prendre en compte cette géométrie de la plantation, la maille élémentaire du couvert (fig 6) a été discrétisée en cellules de petites dimensions $(30 \mathrm{~cm}$ respectivement dans les deux directions horizontales et $20 \mathrm{~cm}$ pour la hauteur), soit plus de 20000 cellules non vides. Cette structuration menant ì des contraintes trop importantes en termes de temps de calcul et stockage mémoire, nous avons utilisé la version 3D simplifiée du modèle Sinoquet : celle-ci ne prend en compte la redispersion du rayonnement par les feuilles que de manière simplifiée, en utilisant des facteurs de correction pour les coefficients dextinction (approximation de Goudriaan, 1977).

$$
K^{\prime}(\Omega)=\sqrt{ } 1-\mathrm{r}-\mathrm{t} \times \mathrm{K}(\Omega)
$$

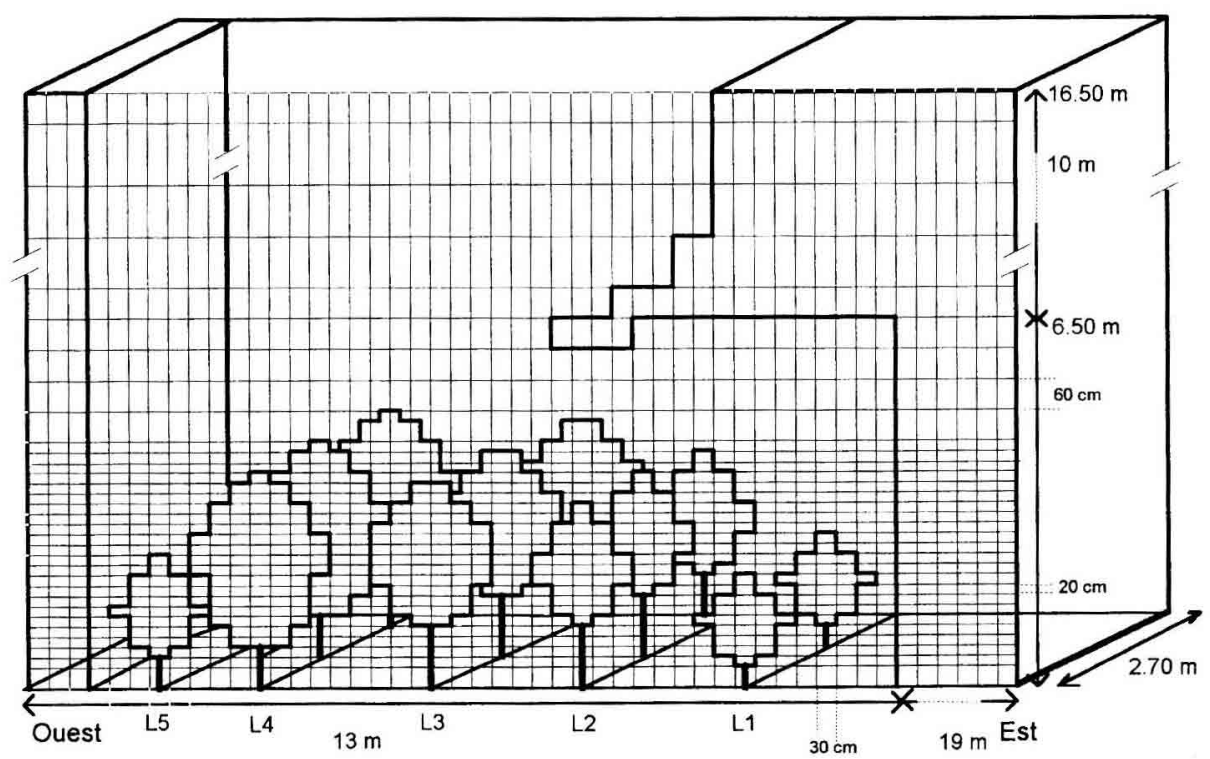

Fig 6. Représentation du maillage élémentaire du couvert. 
où $\mathbf{r}$ et $\mathbf{t}$ sont respectivement les réflectances et transmittances des feuilles dans le visible. Elles ont été mesurées grâce à un capteur PAR sur 20 feuilles de hêtres situées en conditions d'éclairement différentes, ainsi que sur 20 feuilles de charme de l'abri latéral. Tournebize et Sinoquet (1995), ayant précisément testé les différences entre les versions initiales et simplifiées du modèle, concluent à l'absence de différences significatives entre celles-ci lorsque la structuration du couvert est relativement fine.

\section{RÉSULTATS}

\section{Structure du couvert}

La relation allométrique établie à l'échelle de la branche est la suivante :

$$
\begin{aligned}
\mathrm{SFb} & =101,2 \mathrm{D}^{1,17} \mathrm{~L}^{0,76} \\
R^{2} & =0,82 \quad n=80
\end{aligned}
$$

où $\mathrm{Sfb}$ est la surface foliaire de la branche $\left(\mathrm{cm}^{2}\right)$, D le diamètre feuillé $(\mathrm{cm}), \mathrm{L}$ la longueur feuillée $(\mathrm{cm})$.
Dans une deuxième étape, les résultats concernant la surface foliaire des 20 arbres échantillonnés ont permis d'établir une relation allométrique à l'échelle de l'arbre d'une très bonne qualité prédictive (fig 7). L'observation des résidus ne montre pas de biais entre lignes. La relation obtenue s'avère parfaitement cohérente puisque la surface foliaire totale de l'arbre est globalement proportionnelle à sa surface terrière (et donc à sa surface d'aubier) :

$$
\mathrm{SF}=0,0029 \mathrm{D}^{2.08} \quad R^{2}=0,96
$$

où SF est la surface foliaire totale de l'arbre $\left(\mathrm{m}^{2}\right)$, et D son diamètre à $20 \mathrm{~cm}(\mathrm{~mm})$.

Les résultats concernant la distribution d'inclinaison des feuilles (tableau I) mettent en évidence la très faible inclinaison des feuilles de hêtre par rapport à l'horizontale : bien que les feuilles du tiers supérieur soient légèrement plus érigées que celles du bas, la distribution observée reste très proche d'une distribution planophile.

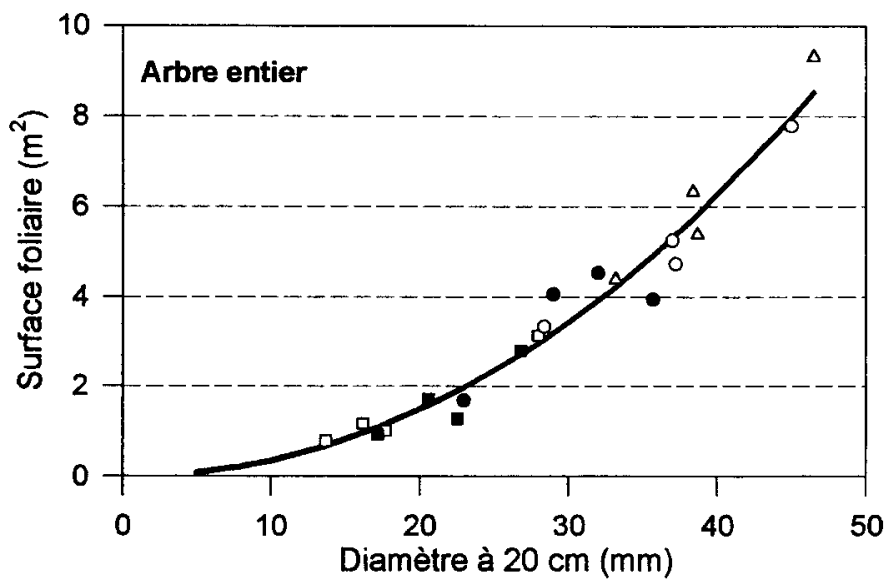

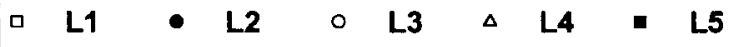

Fig 7. Relation entre la surface foliaire totale d'un hêtre et son diamètre à $20 \mathrm{~cm}$, pour les cinq lignes de la plantation

$$
\text { Régression établie : } \mathrm{SF}=0,0029 \mathrm{D}^{2,08} ; R^{2}=0,96
$$


Tableau I. Distribution d'inclinaison foliaire selon la position des feuilles dans le houppier (fréquence par classe, en $\%$ ).

\begin{tabular}{lcccccc}
\hline & \multicolumn{5}{c}{ Inclinaison/horizontale } \\
\cline { 2 - 5 } & $0-15^{\circ}$ & $15-30^{\circ}$ & $30-45^{\circ}$ & $45-60^{\circ}$ & $60-75^{\circ}$ & $75-90^{\circ}$ \\
\hline Tiers inférieur du houppier & 55 & 31 & 12 & 2 & 0 & 0 \\
Tiers médian & 28 & 44 & 19 & 9 & 0 & 0 \\
Tiers supérieur & 21 & 42 & 23 & 10 & 2 & 2 \\
\hline
\end{tabular}

\section{Mesures de rayonnement et test du modèle Sinoquet}

Pour chacune des deux journées types reconstituées, on compare les données de rayonnement observées et celles issues du modèle, d'une part en termes de proportion de rayonnement transmis (\%) par rapport au rayonnement incident, d'autre part en termes d'énergie transmise cumulée sur chacune de ces journées $\left(\mathrm{MJ} \cdot \mathrm{m}^{-2}\right)$ (grâce à la relation montrée par Varlet-Grancher et al (1981) : 1W de rayonnement global $=2,02 \mu \mathrm{mol} \cdot \mathrm{s}^{-1} \mathrm{de}$ PAR).

\section{Comparaison des fractions de rayonnement incident transmises au sommet et au bas des lignes}

En situation de jour couvert, la proportion de rayonnement transmise au sommet des lignes est très stable, comme attendu (fig 8).

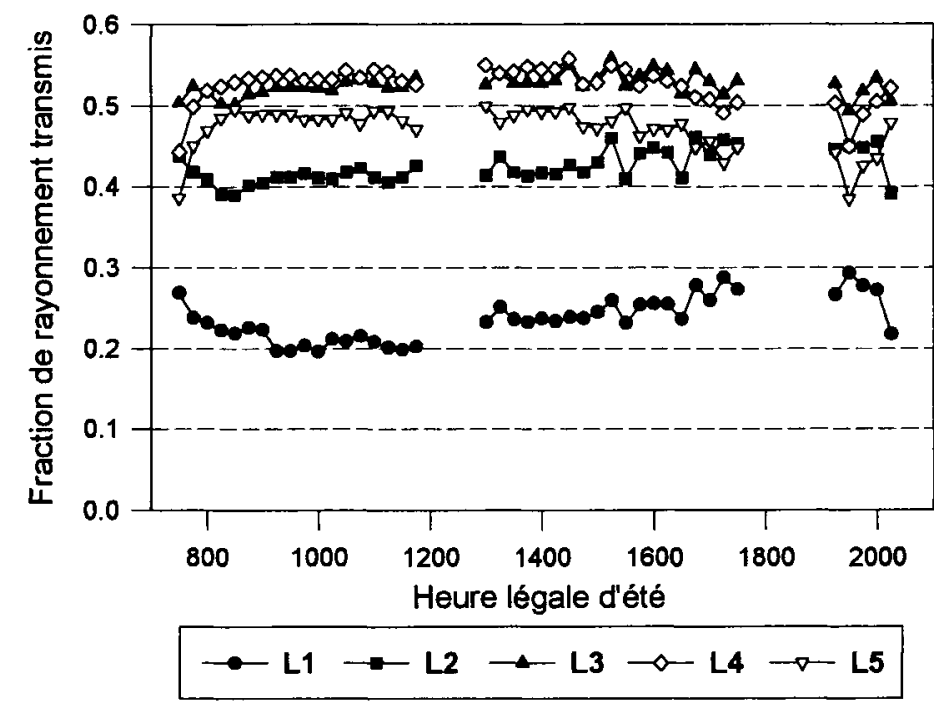

Fig 8. Évolution au cours d'une journée couverte, de la fraction de rayonnement transmis (PAR) au sommet des lignes. Le calcul est effectué avec un pas de temps de 15 min, en rapportant le rayonnement moyen transmis à l'endroit étudié, au rayonnement moyen reçu à découvert pendant la même période de $15 \mathrm{~min}$. 


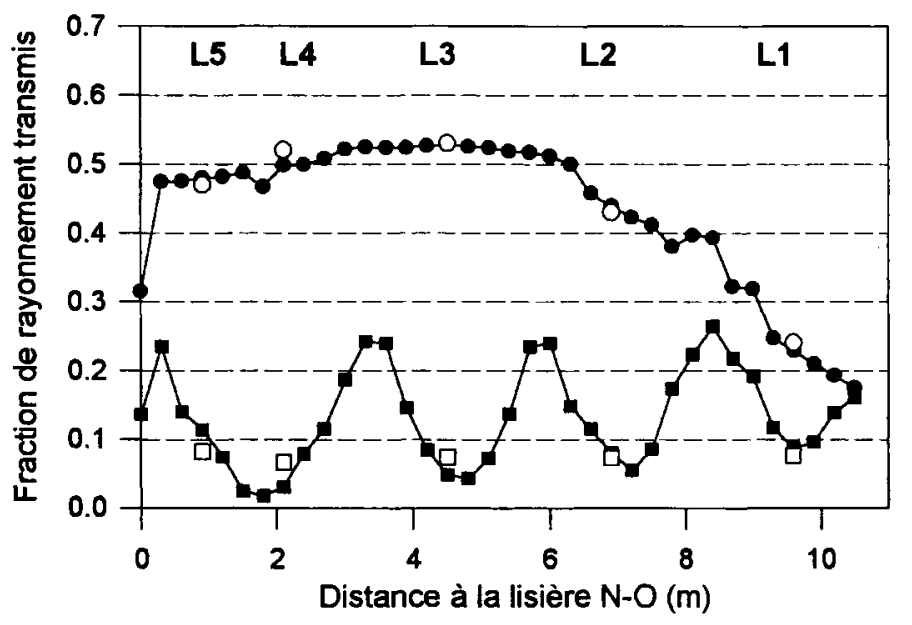

\begin{tabular}{lll|}
$\longrightarrow-4.70 \mathrm{~m}$ - Modèle & $\circ$ & $4.70 \mathrm{~m}$-Mesures \\
$\longrightarrow 0.20 \mathrm{~m}$ - Modèle & $\square$ & $0.20 \mathrm{~m}$-Mesures
\end{tabular}

Fig 9. Variations spatiales de la fraction de rayonnement transmis (PAR) au sommet et au bas des lignes, en journée couverte.

Elle a toutefois tendance à diminuer au cours de la journée pour les capteurs situés côté ouest (L5, L4), et à augmenter pour ceux situés côté est ( $\mathrm{Ll}, \mathrm{L} 2)$, ce qui s'explique vraisemblablement par une répartition différentielle du rayonnement diffus dans la zone proche du disque solaire. Une valeur moyenne du pourcentage de transmission associé à chaque ligne (sommet puis bas) a donc été retenue, à partir d'un nombre d'observations (positions du soleil) également réparties de part et d'autre de l'axe de la plantation. Cette valeur moyenne est ensuite confrontée aux résultats apportés par le modèle (fig 9) : la très bonne adéquation observée en situation de ciel couvert traduit, de la part du modèle, une prise en compte satisfaisante de la géométrie de la plantation.

Lors d'une journée ensoleillée, la figure 10 illustre, pour chaque ligne, l'évolution horaire de la fraction de rayonnement transmis. Là encore, le modèle prédit très cor- rectement l'évolution du rayonnement transmis au sommet des lignes. Les différences sont plus accentuées au niveau du sol, surtout pour la ligne 3 , où la modélisation conduit à une sous-estimation du rayonnement transmis.

\section{Comparaison de l'énergie transmise au sommet et au bas des lignes}

La variabilité spatiale du rayonnement pouvant être une cause d'écarts importants entre les données observées et celles prévues par le modèle, la figure 11 a été établie à partir du pourcentage de transmission moyen et de son écart type sur les neuf cellules $\left(0,81 \mathrm{~m}^{2}\right)$ entourant la position théorique $\mathrm{du}$ capteur. Ces résultats sont ensuite intégrés en termes d'énergie transmise pour les deux journées type (couverte et ensoleillée).

Concernant l'énergie transmise au sommet des lignes (fig 1 la), l'écart relatif entre modèle et mesures est le plus souvent infé- 

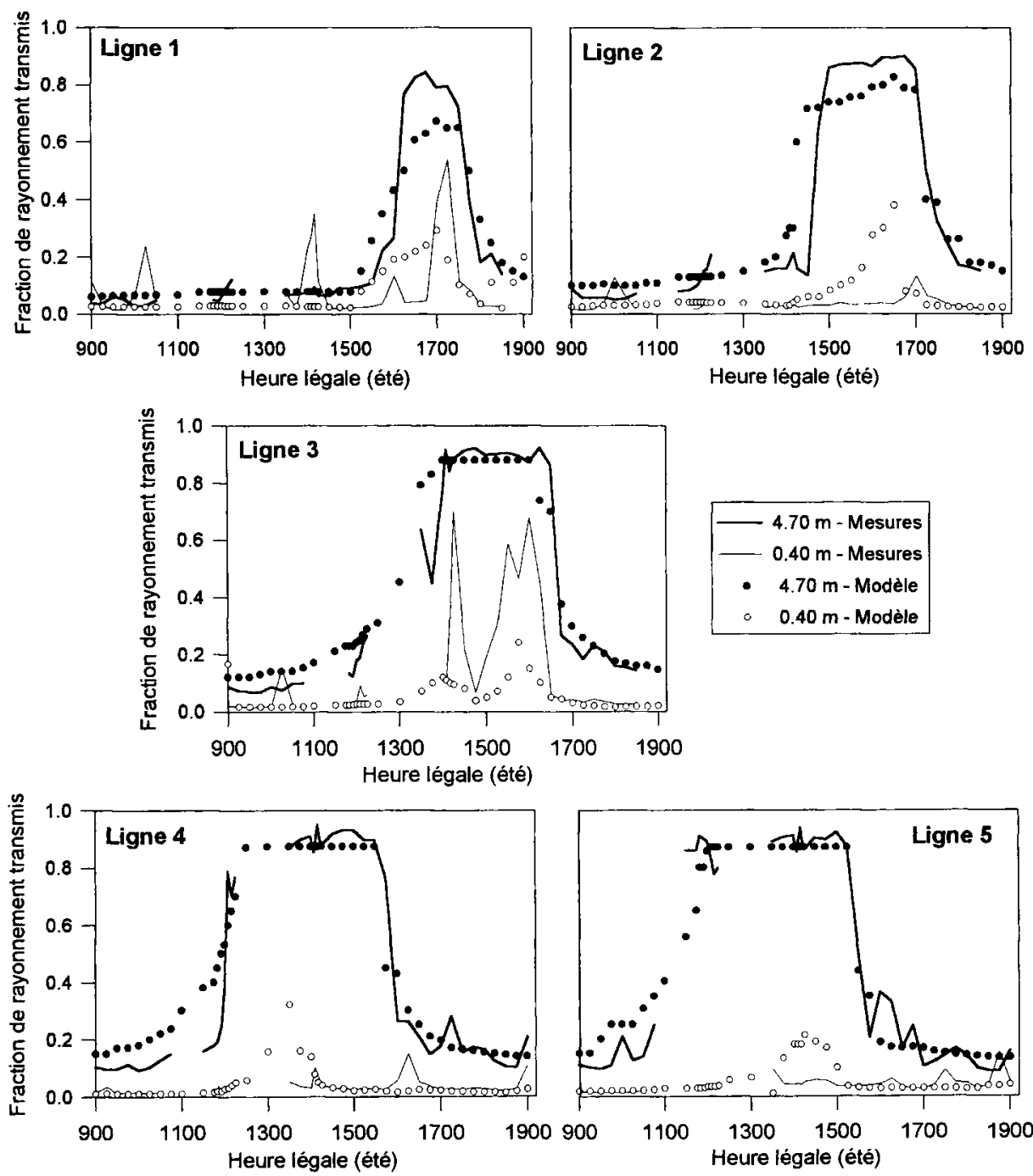

Fig 10. Comparaison des données du modèle et des mesures au cours de la journée type ensoleillée : évolution de la fraction de rayonnement transmis (PAR) au sommet et au bas des lignes.

rieur à $5 \%$, les mesures étant comprises dans l'intervalle formé par la moyenne et l'écart type du rayonnement calculé sur $0,81 \mathrm{~m}^{2}$. On peut considérer que le modèle traduit très correctement, en journée ensoleillée comme en journée couverte, l'ombrage des bandes latérales de forêt sur la plantation.

Les écarts relatifs observés dans l'énergie transmise au sol (fig 11 b) sont beaucoup plus importants, surtout en journée ensoleillée où ils peuvent dépasser $50 \%$ 
a. Sommet des lignes
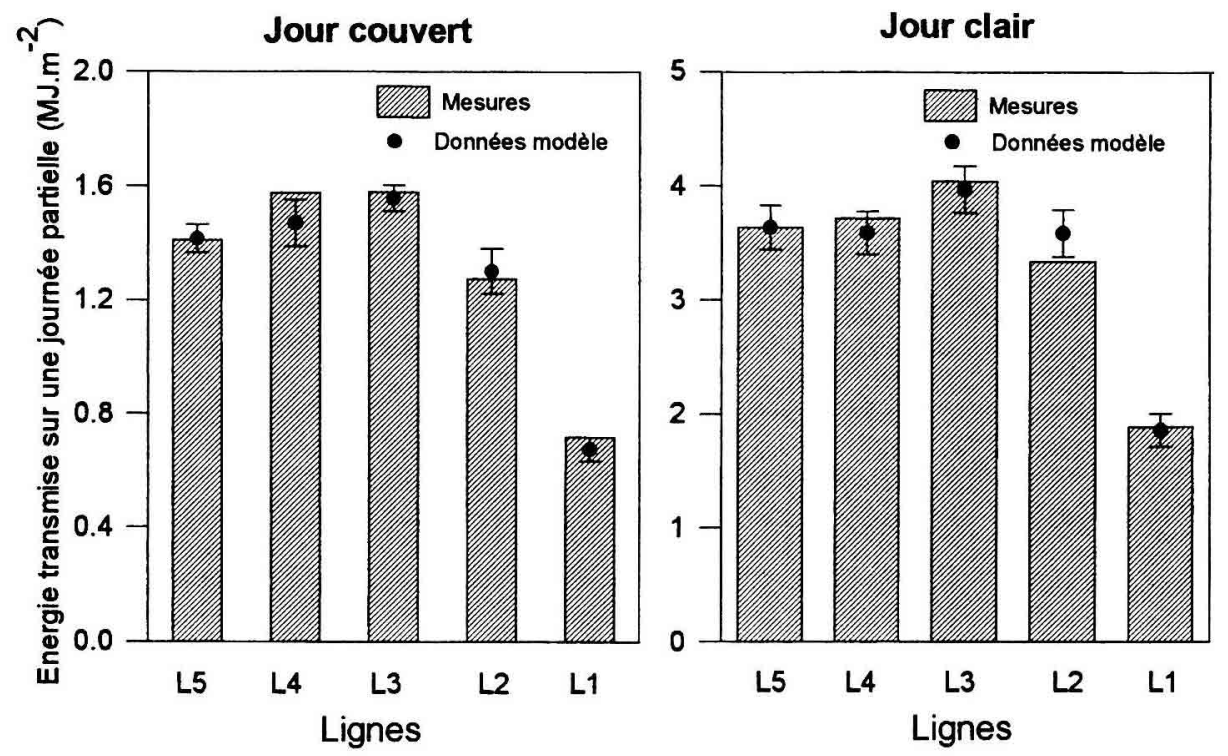

b. Bas des lignes

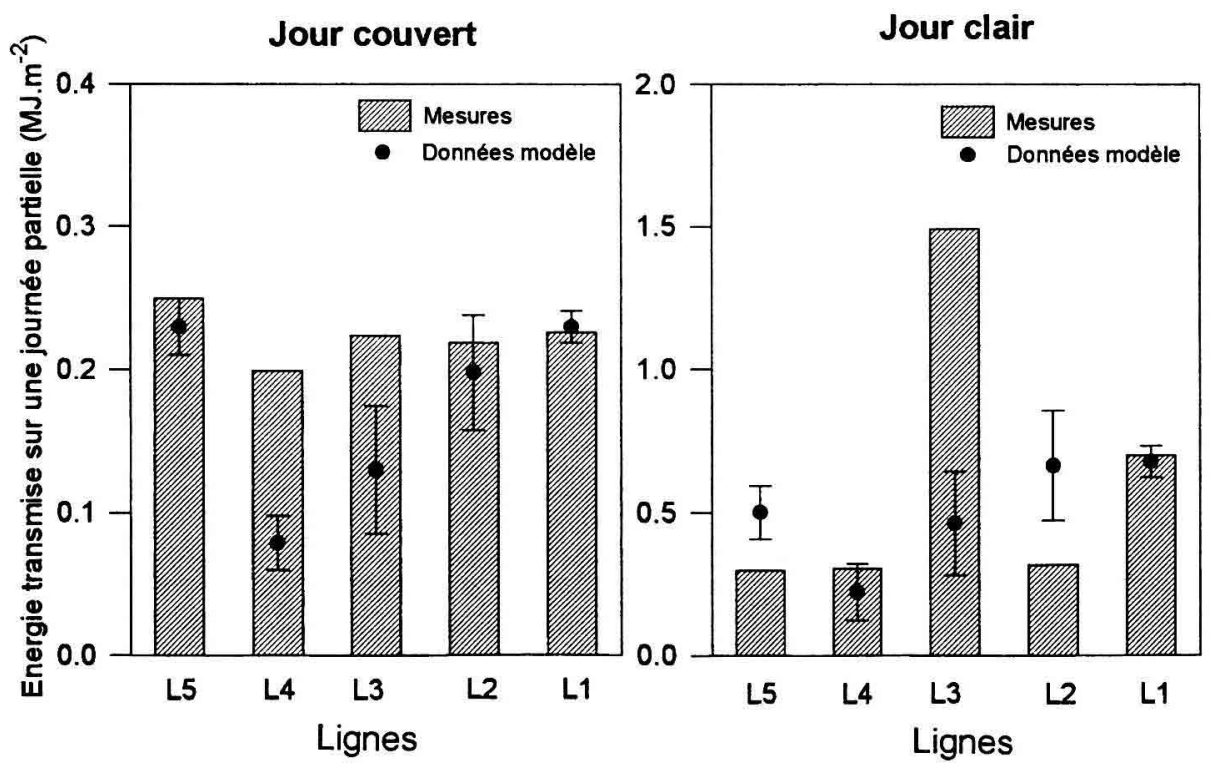

Fig 11. Comparaison entre données du modèle et mesures, en termes d'énergie transmise (PAR) au cours des deux journées types reconstituées (moyenne et écart type calculés sur neuf cellules). 
(ligne 3). Les très fortes variations spatiales du rayonnement transmis au sol en situation ensoleillée (coefficient de variation calculé sur neuf cellules proche de $50 \%$ ) relativisent l'intérêt de ces données, et conduisent davantage à exploiter les résultats obtenus en journée couverte (situation où la variabilité spatiale et temporelle du rayonnement est moindre) : en journée couverte, le modèle conduit, pour les lignes 3 et 4 , à une sous-estimation sensible du rayonnement transmis au sol $(-40$ et $-60 \%$ respectivement). Il est intéressant de noter que, de la ligne 1 à la ligne 4, l'augmentation progressive du volume et de la surface foliaire des houppiers a pour conséquence une diminution progressive du rayonnement au sol estimé par le modèle, alors que les données observées sont, quant à elles, très proches d'une ligne à l'autre.

\section{Caractérisation du microclimat lumineux de la plantation}

L'utilisation du modèle Sinoquet a finalement permis de quantifier la fraction de rayonnement qui, au cours d'une journée de juin, est transmise à l'apex de l'arbre moyen de chaque ligne (et non à $4,70 \mathrm{~m}$ ). La figure 12 montre que la présence de l'abri latéral se traduit globalement par une diminution de 50 à $80 \%$ de l'énergie incidente. Côté est, la présence des branches de la lisière, qui empiètent sur la plantation, handicape lourdement la ligne 1, laquelle reçoit environ moitié moins de lumière que la ligne 5.

Des différences significatives sont cependant à signaler entre la journée couverte et la journée ensoleillée :

- en journée couverte, la ligne 3 est la plus favorisée, car plus proche de l'axe central de la plantation, mais les différences sont finalement assez modestes entre les lignes 2 , 3,4 , et 5 : la ligne 2 (la moins avantagée), reçoit, par rapport à la ligne $3,17 \%$ seulement de rayonnement en moins.

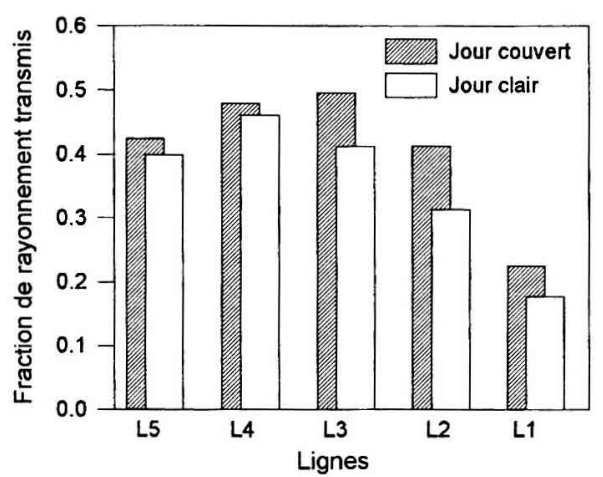

Fig 12. Fraction de rayonnement transmis (PAR) au sommet de l'arbre moyen de chaque ligne. Valeur calculée sur une journée entière de juin, en tenant compte de l'évolution horaire du rayonnement incident.

- en journée ensoleillée, on observe une répartition dissymétrique du rayonnement dans la plantation, due à l'orientation des lignes $\left(38^{\circ}\right.$ par rapport à l'axe nord-sud) : la ligne 4 est cette fois la plus avantagée, sa position excentrée côté ouest lui permettant de profiter du rayonnement direct dans le créneau horaire où l'énergie solaire est la plus forte (de -2 heures à +2 heures par rapport au midi solaire). Les écarts entre lignes sont ainsi plus marqués qu'en situation de ciel couvert : la ligne 2 , éclairée relativement tard dans la journée, reçoit seulement $68 \%$ du rayonnement disponible pour la ligne 4 .

\section{DISCUSSION}

\section{Test du modèle et caractérisation de la structure de l'abri latéral}

Si le test du modèle apporte des résultats très satisfaisants en regard du petit nombre de données recueillies, il faut souligner que cette démarche reste partielle, compte tenu des fortes variations spatiales de rayonnement au sol, ainsi que des vraisemblables 
variations structurales de l'abri latéral d'un endroit à l'autre de la plantation. L'utilisation d'un nombre plus important de capteurs, positionnés par exemple à mi-distance entre les lignes, aurait sans aucun doute permis d'améliorer la qualité du test du modèle. Une validation plus complète du modèle aurait de plus nécessité l'acquisition de données de rayonnement sur plusieurs placettes successives, acquisitions répétées au cours de la saison de végétation.

Malgré tout, le test du modèle a montré la qualité de l'estimation fournie pour le rayonnement transmis au sommet des lignes de la plantation : l'approche adoptée pour la géométrie des bandes latérales de forêt a suffi pour traduire très correctement, dans la maille du modèle, l'ombrage dû à celles-ci. La très grande souplesse du modèle Sinoquet, dans sa capacité à prendre en compte la complexité d'une structure végétale donnée (ici l'abri latéral et sa lisière) permet d'expliquer la remarquable adéquation observée entre données issues du modèle et données observées.

\section{Caractérisation de la structure de la plantation}

La méthode utilisée pour estimer la surface foliaire des jeunes arbres de la plantation s'est révélée très satisfaisante, dans la mesure où les relations de régression établies montrent un coefficient de corrélation élevé, et ne font apparaître aucun biais entre lignes. Ces résultats confortent ceux établis sur de jeunes hêtres par Lemée (1987), lequel soulignait alors l'étroitesse des relations liant l'appareil foliaire à la charpente ligneuse.

Les résultats obtenus concernant le rayonnement transmis au sol (tableau II version 1) comportent une cohérence intéressante à discuter, puisque l'écart entre mesures et estimation du modèle semble d'autant plus important que les arbres sont développés.
Tableau II. Rayonnement transmis (PAR) au bas des lignes en journée couverte : écart relatif des résultats du modèle (versions 1, 2, et 3) par rapport aux mesures (\%).

\begin{tabular}{lrrrrr}
\hline & $L 5$ & $L 4$ & $L 3$ & $L 2$ & $L 1$ \\
\hline Modèle version 1 & -5 & -60 & -41 & -10 & $+2,6$ \\
Modèle version 2 & +60 & -10 & +20 & +60 & +50 \\
Modèle version 3 & +3 & -25 & -5 & +1 & +8
\end{tabular}

Modèle version 1 : densité foliaire uniforme dans les houppiers; pas d'agrégation. Modèle version 2 : densité foliaire uniforme ; paramètre d'agrégation égal à 0,6 . Modèle version 3 : densité foliaire 4 fois plus faible dans les parties 1 et 3 , et sur les bords des houppiers ; pas d'agrégation.

L'hypothèse d'un biais dans la régression établie, menant à une surestimation de la surface foliaire pour les diamètres élevés, peut être raisonnablement écartée, compte tenu des raisons évoquées ci-dessus. Il serait en effet nécessaire, pour observer un rayonnement transmis sous la ligne 4 égal à la valeur mesurée, d'envisager une surestimation de la surface foliaire de plus de $60 \%$.

En revanche, il paraît pertinent de discuter l'hypothèse retenue par le modèle d'un agencement aléatoire des feuilles dans les houppiers. À la différence des conifères où l'agrégation des aiguilles au sein des pousses doit impérativement être prise en compte par un paramètre d'agencement (Whitehead et al, 1990 ; Oker-Blom et Kellomaki, 1983), les travaux consacrés aux couverts feuillus montrent que l'hypothèse d'un agencement aléatoire des feuilles est parfois vérifiée (Walter, 1993), mais souvent mise en défaut (Neuman et al, 1989 ; Baldocchi et Hutchinson, 1986 ; Cohen et Fuchs, 1987). Le processus d'agrégation conduit, du fait d'un auto-ombrage plus marqué au sein des feuilles, à une interception de rayonnement plus faible, et assure donc une meilleure pénétration du rayonnement dans les houppiers (Oker-Blom et Kellomäki, 1983). 
Afin de tester cette hypothèse, nous avons étudié les résultats fournis par le modèle Sinoquet après introduction pour les cinq lignes de la plantation d'un paramètre d'agencement de 0,6 (tableau II version 2) : si l'écart entre données du modèle et mesures est considérablement réduit pour la ligne 4 , on observe en revanche une forte surestimation du rayonnement sous les lignes 1, 2 et 5 . L'hypothèse de l'agrégation des feuilles n'est donc pas à elle seule suffisante, car elle conduit nécessairement à la prise en compte d'une agrégation forte sur les arbres les plus développés, et moins importante sur de petits houppiers. Or l'observation empirique de la morphologie des arbres amène plutôt à considérer une agrégation faible pour les houppiers les plus développés des lignes 3 et 4 (où l'importance de la ramification semble assurer une répartition plus homogène des feuilles), et plus forte pour les petits houppiers des lignes 1 et 5 (où les feuilles semblent groupées au niveau des quelques branches existantes).

Enfin, des travaux récents ont mis en évidence de fortes variations spatiales dans la densité de surface foliaire au sein des houppiers (Wang et al, 1990 ; Whitehead et al, 1990 ; Webb et Ungs, 1993) et remettent en cause l'hypothèse classiquement retenue (car pratique) d'une répartition uniforme de la densité de surface foliaire. Dans le cas qui nous intéresse, la distinction des trois parties (strates) P1, P2, P3 permet d'envisager une densité de surface foliaire plus faible au sommet du houppier (P1), où les branches sont plus jeunes et moins ramifiées, ainsi qu'à la base du houppier (P3), où les branches, plus âgées, se trouvent en phase d'élagage. Il paraît d'autant plus cohérent d'envisager l'existence d'un tel gradient, que de nombreux travaux, portant certes sur des conifères (Halldin, 1985 ; Beadle et al, 1982 ; Wang et al, 1990), en montrent clairement l'existence. De la même manière, on peut raisonnablement envisager l'existence d'un gradient horizontal fonction de la distance à l'axe principal : en effet, la partie apicale des branches est peu ramifiée, et porte moins de feuilles que les parties plus anciennes et plus ramifiées. Lorsque la branche est suffisamment âgée, un début d'élagage apparaît, et la densité foliaire maximale se trouve alors dans la partie médiane de la branche.

S'il paraît difficile de quantifier de manière rigoureuse les conséquences de ces deux gradients en termes de transmission du rayonnement, les résultats fournis par le modèle lorsqu'on modifie la répartition spatiale des feuilles illustrent bien l'importance de ce facteur: pour chaque arbre des lignes 1 à 5 , on a introduit dans les strates 1 et 3 des houppiers (hauteurs relatives issues des mesures faites in situ), ainsi que dans toute la couche la plus externe de cellules, une densité foliaire 4 fois inférieure à celle de l'intérieur de la strate 2 du houppier (la surface foliaire totale restant la même). Dans ces conditions, on observe (tableau II version 3) une augmentation du rayonnement transmis au sol, qui, sur toutes les lignes, conduit à des écarts modestes avec les mesures réalisées, à l'exception de la ligne 4 où la différence s'élève encore à $25 \%$. Ces résultats illustrent bien le fait que le rayonnement reçu au sol dépend largement des possibilités de transmission sur le bord des houppiers, directement ou après traversée d'une épaisseur limitée de feuilles : la répartition spatiale des feuilles au sein des houppiers, et, plus généralement, la forme et les dimensions choisies pour les houppiers, sont donc, vis-à-vis de l'interception du rayonnement, des facteurs cruciaux dont l'importance a déjà été soulignée dans d'autres travaux (Wang et al, 1990 ; Kuuluvainen et Pukkala, 1987).

\section{Intérêts et limites de l'approche issue de modélisation}

La principale limite à l'approche utilisée ici est donc la nécessité d'une bonne caractérisation de la structure du couvert étudié : 
géométrie du peuplement, répartition spatiale et agencement des feuilles sont autant de données difficiles à acquérir, mais indispensables si l'on souhaite quantifier la répartition de la lumière au sein de structures discontinues. Par ailleurs, il faut souligner que cette caractérisation imparfaite de la structure du peuplement ne conduit, en termes de répartition du rayonnement, qu'à une erreur modeste, puisqu'elle n'excède pas, dans notre cas, $5 \%$ du rayonnement incident.

Lorsque cette caractérisation structurale est correctement réalisée, la modélisation permet alors d'envisager une caractérisation du microclimat lumineux en tout point du couvert, quelle que soit la saison envisagée : la qualité des estimations apportées par le modèle Sinoquet en fait donc un outil d'un grand intérêt pour la caractérisation du microclimat lumineux des couverts forestiers complexes. Il permet par ailleurs de déterminer l'énergie lumineuse absorbée par l'ensemble ou par une partie du couvert, et constitue donc un outil méthodologique important dans l'étude de l'effet de la lumière sur la croissance des jeunes peuplements. C'est cette dernière approche du modèle que nous développerons prochainement.

\section{REMERCIEMENTS}

Nos plus sincères remerciements vont à B PilardLandeau et J Piat (ONF-Stir Nord-ouest) pour leur aide précieuse dans la réalisation de cette étude. Notre reconnaissance va également à $\mathrm{H}$ Sinoquet (Inra Clermont-Ferrand) et à R Bonhomme (Inra Grignon) pour leur aide et leurs conseils. Enfin, nous remercions sincèrement les deux lecteurs anonymes.

\section{RÉFÉRENCES}

Baldocchi DD, Hutchinson BA ( 1986) On estimating canopy photosynthesis and stomatal conductance in a deciduous forest with clumped foliage. Tree Physiol 2, 155-168
Beadle CL, Talbot H, and Jarvis PG (1982) Canopy structure and leaf area index in mature Scots pine forest. Forestry 55, 105-123

Charles-Edwards DA, Thorpe MR (1976) Interception of diffuse and direct-beam radiation by a hedgerow apple orchard. Ann Bot 40, 603-613

Cohen S, Fuchs M (1987) The distribution of leaf area, radiation, photosynthesis and transpiration in a Shamouti Orange hedgerow orchard. I. Leaf area and radiation. Agric For Meteorol 40, 123-144

Goudriaan J (1977) Crop micrometeorology: a simulation study. Centre for Agricultural Publishing and Documentation, Wageningen, the Netherlands

Halldin S (1985) Leaf and bark distribution in a pine forest. In : The Forest-Atmosphere Interaction (BA Hutchinson, BBD Hicks, eds), Reidel Publishing Co, 39-58

Jackson JE, Palmer JW (1972) Interception of light by model hedgerow orchards in relation to latitude, time of year and hedgerow configuration and orientation. J Appl Ecol 9, 341-357

Kimes DS, Kirchner JA (1982) Radiative transfer model for heterogeneous 3-D scenes. Appl Optics 19, 2801-2811

Kuuluvainen T, Pukkala T (1987) Effect of crown shape and tree distribution on the spatial distribution of shade. Agric For Meteorol 40,215-231

Lemée G (1987) Dynamique de fermeture par régénération et évolution morphométrique du hêtre dans les vides d'une forêt non exploitée. Bull Ecol 18, 1-11

Lemeur R, Blad BL (1974) A critical review of light models for estimating the shortwave radiation regime of plant canopies. Agric Meteorol 14, 255-286

Neumann HH, den Hartog G, and Shaw RH (1989) Leaf area measurements based on hemispheric photographs and leaf-litter collection in a deciduous forest during autumn leaf-fall. Agric For Meteorol $45,325-345$

Oker-Blom P, Kellomäki S (1983) Effect of grouping of foliage on the within-stand and within-crown light regime: comparison of random and grouping canopy models. Agric Meteorol 28, 143-155

Pontailler J-Y (1990) A cheap quantum sensor using a gallium arsenide photodiode. Functional Ecology 4 , $591-596$

Sinoquet H (1988) Modélisation des échanges radiatifs de courte longueur d'onde dans certains couverts hétérogènes. Thèse, Ina-PG, $80 \mathrm{p}$

Sinoquet $H$ (1993) Modelling radiative transfer in heterogeneous canopies and intercropping systems. In : Crop Structure and Light Microclimate (C VarletGrancher, R Bonhomme, H Sinoquet, eds), Inra Paris, 207-228

Tournebize R, Sinoquet H (1995) Light interception and partitioning in a shrub/grass mixture. Agric For Meteorol 3-4, 277-294

Varlet-Grancher C, Chartier M, Gosse G, Bonhomme R (1981) Rayonnement utile pour la photosynthèse 
des végétaux en conditions naturelles : caractérisation et variations. Acta Oecol Plant 16, 3-26

Walter JMN (1993) Canopy geometry and the interception of PAR in a temperate deciduous forest: an interpretation of hemispherical photographs. In : Crop Structure and Light Microclimate, (C Varlet-Grancher, R Bonhomme, H Sinoquet, eds), Inra Paris, 373-386

Wang YP, Jarvis PG (1990) Description and validation of an array model-MAESTRO. Agric For Meteorol 51, 257-280
Wang YP, Jarvis PG, Benson ML (1990) Twodimensional needle area density distribution within the crowns of Pinus radiata. For Ecol Manag 32, 217-237

Webb WL, Ungs MJ (1993) Three-dimensional distribution of needle and stem surface area in a Douglas-fir. Tree Physiol 13, 203-212

Whitehead D, Grace JC, Godfrey MJS (1990) Architectural distribution of foliage in individual Pinus radiata D Don crowns and the effects of clumping on radiation interception. Tree Physiol 7, 135-155 